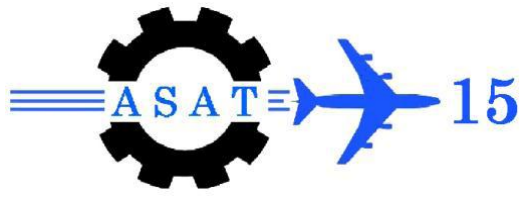

\title{
Smart Identification of Overlapping Strip Pairs/Regions for Optimized LiDAR System Calibration
}

\author{
E. Hamza ${ }^{*}$ and A.Habib ${ }^{\dagger}$
}

\begin{abstract}
Recently, laser scanning systems, onboard airborne and terrestrial mobile mapping systems, have been established as a leading technology for collecting high density 3D information from an object's surface. The availability of generated surface models is very important for various industrial, military, environmental, and public applications. The accuracy of the derived point cloud coordinates from a LiDAR system is affected by inherent systematic and random errors. The impact of random errors depends on the precision of the system's measurements, which comprise position and orientation information from the GPS/INS unit, mirror angles, and ranges. On the other hand, systematic errors are mainly caused by biases in the mounting parameters (i.e., lever arm offset and boresight angles) relating the system components as well as biases in the system measurements (e.g., ranges and mirror angles). In order to ensure the geometric quality of the collected point cloud, the LiDAR systems should undergo a rigorous calibration procedure to estimate the system parameters that minimize the discrepancies between conjugate surface elements in overlapping LiDAR strips. The main objective of this paper is to look into an existing LiDAR system calibration technique, which is based on manual selection of overlapping regions between LiDAR strips and how to increase the efficiency of this technique by automatic selection of appropriate overlapping strip pairs, which should achieve the minimum optimal flight configuration that maximizes the impact of the discrepancies among conjugate surface elements in overlapping strips as well as automatic selection of regions within the appropriate overlapping strip pairs. The methodology of the proposed technique can be summarized as follows: first, the LiDAR strip pairs are grouped based on the flight configuration; second, appropriate overlapping strip pairs from each group is automatically selected; third, regions within the appropriate overlapping strip pairs are automatically selected based on their angles (slopes and aspects) and distribution; finally, the calibration procedure is applied. The experimental results have shown that the quality of the estimated parameters using the automatic selection are quite comparable to the estimated parameters using the manual selection while the proposed method is fully automated, and much faster.
\end{abstract}

Keywords: LiDAR; Laser Scanning; Calibration.

\section{Introduction}

Over the past few years, laser scanning systems onboard airborne and terrestrial mobile mapping systems have been established as a leading technology for the acquisition of high density 3D spatial data. The availability of 3D surface data is very important for several

\footnotetext{
Dep. of Geomatics Engineering, University of Calgary, 2500 University Drive NW, Calgary, Alberta, T2N 1N4, Canada, ehhamza@ucalgary.ca

$\dagger$ Dep. of Geomatics Engineering, University of Calgary, 2500 University Drive NW, Calgary, Alberta, T2N 1N4, Canada, ahabib@ucalgary.ca
} 
applications such as environmental monitoring, military simulation, contour mapping, transportation planning, oil and gas exploration, mining, shoreline management, 3D city modeling, and forest mapping. The ability of the LiDAR system to determine 3D points on the ground is the result of multiple components integrated to work together as shown in Figure 1. The Global Positioning System (GPS) provides the position of the sensor during the mission. An Inertial Navigation System (INS) is used to provide the sensor attitude, commonly referred to as roll, pitch, and heading. The laser scanner records the scan angle relative to the sensor platform in addition to estimating the distance from the sensor to the ground point by measuring the time delay between a laser pulse transmission and its detection. The above information allows for the determination of the location of the points along the mapped surface.

The accuracy of the derived point cloud coordinates from a LiDAR system is affected by inherent systematic and random errors. The impact of random errors depends on the precision of the system's measurements, which comprise position and orientation information from the GPS/INS unit, mirror angles, and ranges. On the other hand, systematic errors are mainly caused by biases in the mounting parameters (i.e., lever arm offset and boresight angles) relating the system components as well as biases in the system measurements (e.g., ranges and mirror angles).

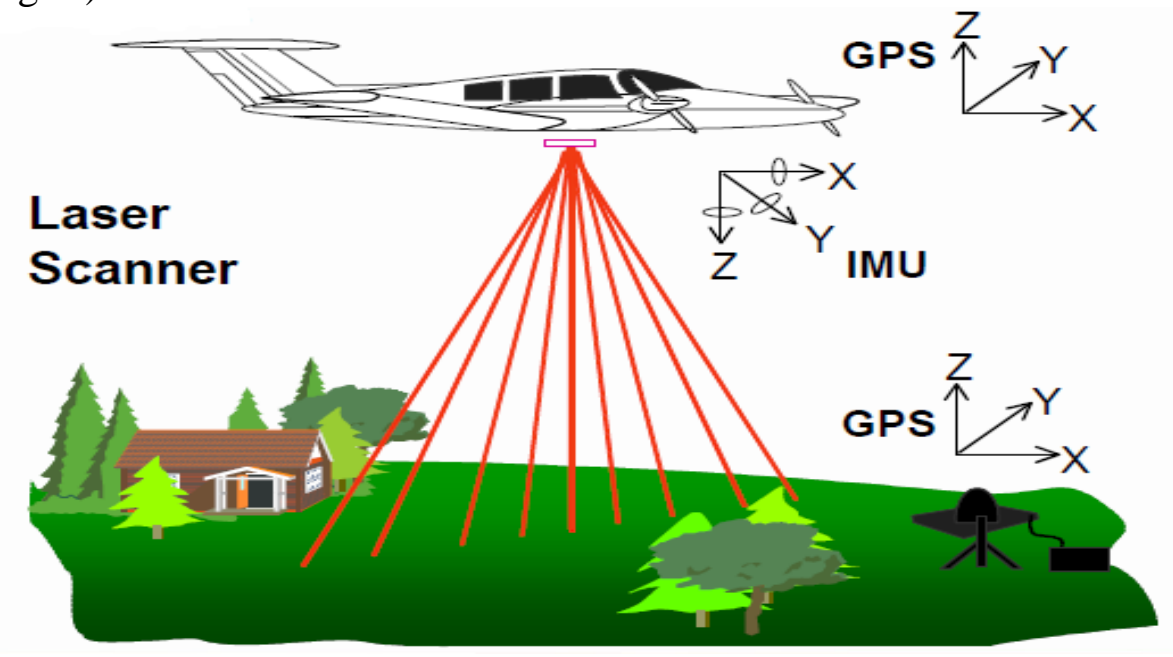

Figure 1. In-flight LiDAR system components

In order to ensure the geometric quality of the collected point cloud, the LiDAR systems should undergo a rigorous calibration procedure to estimate the system parameters that minimize the discrepancies between conjugate surface elements in overlapping LiDAR strips. LiDAR calibration requires the identification of common elements or primitives in overlapping LiDAR strips as well as control data. Distinct points have been used as a primitive in photogrammetric data for a long time. However, it is well-known that distinct points cannot be directly captured by a LiDAR system as the system produces irregular point data unlike photogrammetric systems (Ackermann, 1999). Planar patches are indirectly identified to be used as conjugate surface elements in overlapping LiDAR strips (Skaloud and Lichti, 2006; Habib et al., 2007; Lee et al., 2007; Skaloud and Schaer, 2007; Habib et al., 2009b). Planar patches such as gable roofs can be extracted by a plane segmentation process. These planar patches can be used as suitable primitives in overlapping LiDAR strips. Also, these planar patches should have varying slope and aspect angles to be beneficial in the LiDAR system calibration. 
Typically, the undertaken steps during the current LiDAR system calibration procedure, which is proposed by Kersting (2011), include manual selection of overlapping strip pairs/regions among the available LiDAR strips as shown in Figure 2.

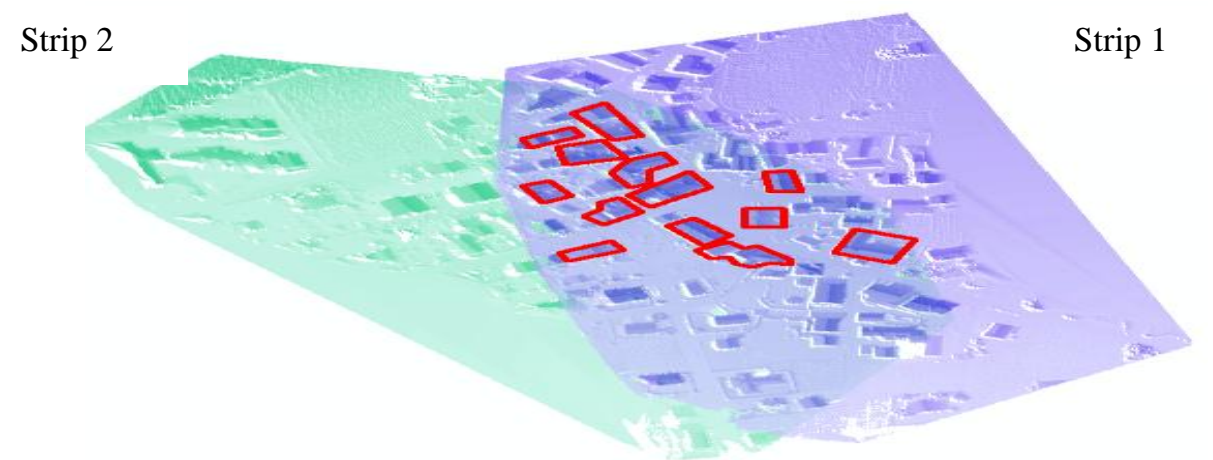

Figure 2. Sample of manually selected pairs/regions within the overlapping area between two LiDAR strips

Some problems could arise when using manually-selected pairs/regions as follows:

- Insufficient configuration will reduce the quality of the estimated parameters,

- Redundant slope and aspect will increase the execution time of the calibration procedure, and

- The manual selection reliance on the experience of the operator.

Thus, these problems will affect the quality of the calibration procedure. The main objective of this paper is to investigate an existing LiDAR system calibration technique, which is based on the manual selection of overlapping regions among LiDAR strips and how to increase the efficiency of this technique as follows: automatic selection of appropriate overlapping strip pairs, which should achieve the minimum optimal flight configuration that maximizes the impact of the discrepancies among conjugate surface elements in overlapping strips. The minimum optimal flight configuration consists of three overlapping strip pairs (two flown in different flying heights in opposite directions and one flown in parallel direction), Kersting (2011). Also, this paper introduces automatic selection of regions within the appropriate overlapping strip pairs. The main criteria for the selection of these regions are as follows: the selected regions should exhibit good variation in the topography (i.e., surfaces with varying slope and aspect values should be utilized). Moreover, these regions should be welldistributed within the overlapping region. The structure of this paper can be summarized as follows: section 2 introduces the proposed methodology for automatic selection of suitable overlapping strip pairs/regions to be used for optimized LiDAR system calibration. Section 3 presents the experiments carried out using a real datasets to demonstrate the comparative analysis between manual and automatic selection of regions among overlapping strip pairs after applying the calibration procedure. Finally, section 4 presents concluding remarks and recommendations for future work.

\section{Proposed Methodology}

As mentioned before, due to the inefficiency of manual selection of overlapping pairs/regions between LiDAR strips, in this paper, a novel method of automatic selection of suitable overlapping strip pairs/regions is introduced for accurate, fast, and reliable LiDAR system calibration. Figure 3 shows the stages of the proposed methodology. A detailed description of the proposed methodology is explained in the following sub-sections. 


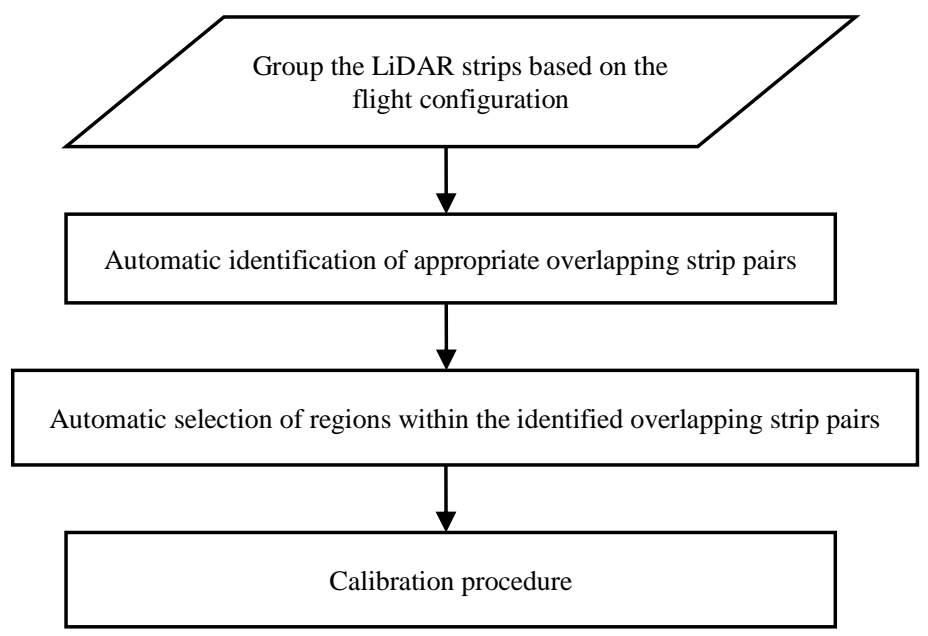

Figure 3. Flow chart of the proposed methodology

\subsection{Group the LiDAR Strips Based on the Flight Configuration}

In this step, the average flying height and flight direction (heading angle) of each LiDAR strip (as shown in Figure 4) are computed. Table 1 presents examples of the average flying height and flight direction of each LiDAR strip. The LiDAR strips that have variations in the average flying heights within a predefined threshold (few meters) are considered to be at the same flying height. Then, the LiDAR strips that have the same flying height are classified based on the flight direction into (parallel/cross/opposite) groups. Table 2 presents examples of different categories and groups of LiDAR strips based on the averaging flying heights and flight directions. From Tables 1 and 2 one can note that, the strips (1, 2, 3, 4, and 9) have the same flying height, denoted as "Category 1 ", and strips $(5,6,7$, and 8) have a different flying height, denoted as "Category 2". In category 1, 10 possible groups (parallel/cross/opposite) between LiDAR strips are automatically identified. Also, in category 2, 6 possible groups between LiDAR strips are automatically identified. Note, 10 and 6 possible pairs are the total number of combinations from the flight lines in each category taken 2 at a time regardless of their order.

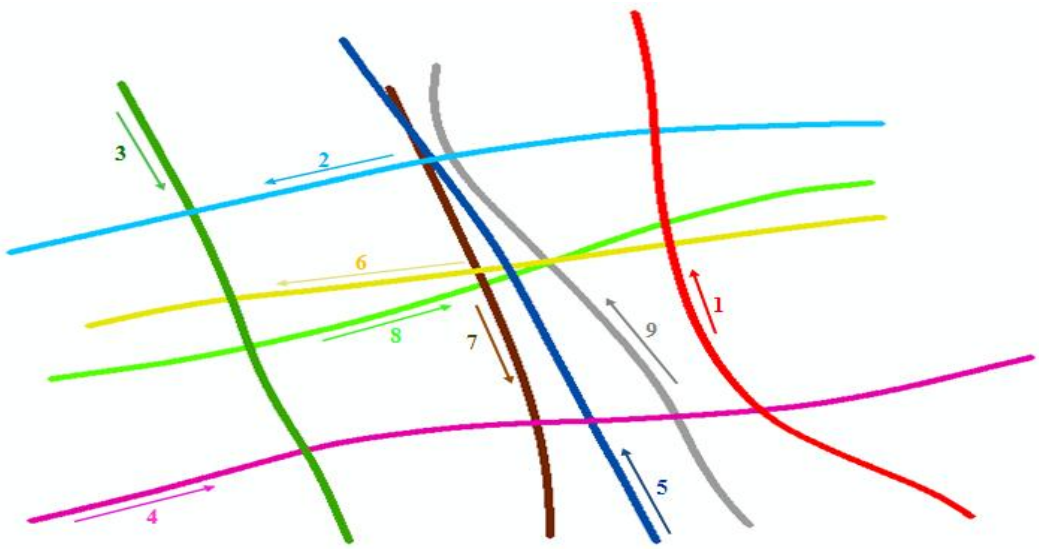

Figure 4. The flight configuration of 9 LiDAR strips 
Table 1. The calculation of the average flying height and flight direction of each LiDAR strip

\begin{tabular}{c|c|c}
\hline \hline Strip NO. & Average flying height (m) & Average flight direction (heading angle) (deg) \\
\hline \hline 1 & 696 & 112 \\
\hline 2 & 698 & -155 \\
\hline 3 & 693 & -90 \\
\hline 4 & 708 & 19 \\
\hline 5 & 796 & 100 \\
\hline 6 & 812 & -172 \\
\hline 7 & 795 & -93 \\
\hline 8 & 779 & 6 \\
\hline 9 & 703 & 137 \\
\hline \hline
\end{tabular}

Table 2. Different categories and groups of LiDAR strips based on the flying heights and their relative flight directions

\begin{tabular}{|c|c|c|c|}
\hline First strip & Second strip & Category based on the flying height & Flight Direction \\
\hline 1 & 9 & \multirow{10}{*}{ Category 1} & Parallel Direction \\
\hline 1 & 3 & & Opposite Direction \\
\hline 2 & 4 & & Opposite Direction \\
\hline 3 & 9 & & Opposite Direction \\
\hline 1 & 2 & & Cross Direction \\
\hline 1 & 4 & & Cross Direction \\
\hline 2 & 3 & & Cross Direction \\
\hline 2 & 9 & & Cross Direction \\
\hline 3 & 4 & & Cross Direction \\
\hline 4 & 9 & & Cross Direction \\
\hline 5 & 7 & \multirow{6}{*}{ Category 2} & Opposite Direction \\
\hline 6 & 8 & & Opposite Direction \\
\hline 5 & 6 & & Cross Direction \\
\hline 5 & 8 & & Cross Direction \\
\hline 6 & 7 & & Cross Direction \\
\hline 7 & 8 & & Cross Direction \\
\hline
\end{tabular}

\subsection{Automatic Identification of Appropriate Overlapping Strip Pairs}

The selection of suitable overlapping strip pairs will be achieved by the following two steps:

- Automatic extraction of the overlapping region between the grouped LiDAR strips

- Automatic identification of appropriate overlapping strip pairs from each group to be used in the calibration procedure.

\subsubsection{Automatic extraction of the overlapping region between the grouped LiDAR strip pairs}

The idea of this method is to project the 3D LiDAR strip points on a $2 \mathrm{D}$ grid. The cell dimension of the gird should be greater than the average point spacing and expected voids in the LiDAR data (Lari et al., 2011). Figure 5 illustrates the flowchart of the algorithm for deriving the overlapping region between two LiDAR strips. First, an empty 2D grid is created for each LiDAR strip. Then, both strips are scanned and the cell in each strip is selected as overlapping cell if both strips have any points inside this cell. Finally, both strips are rescanned and the points within the overlapping cells are only included. These points will represent the common overlapping region between two LiDAR strips. Figure 6 shows the 
selected overlapping region between two LiDAR strips, where the overlapping cells are labeled as $(\sqrt{ })$ and the non-overlapping cells are labeled as $(\mathbf{x})$. Figure 7 shows the visualization of the overlapping area between two LiDAR strips. The overlap percentage is calculated as in Equation 1.

$$
\text { overlap percentage }=\frac{\text { No of points in the overlap area }}{\text { Total number of points in the original LiDAR strip }} \times 100
$$

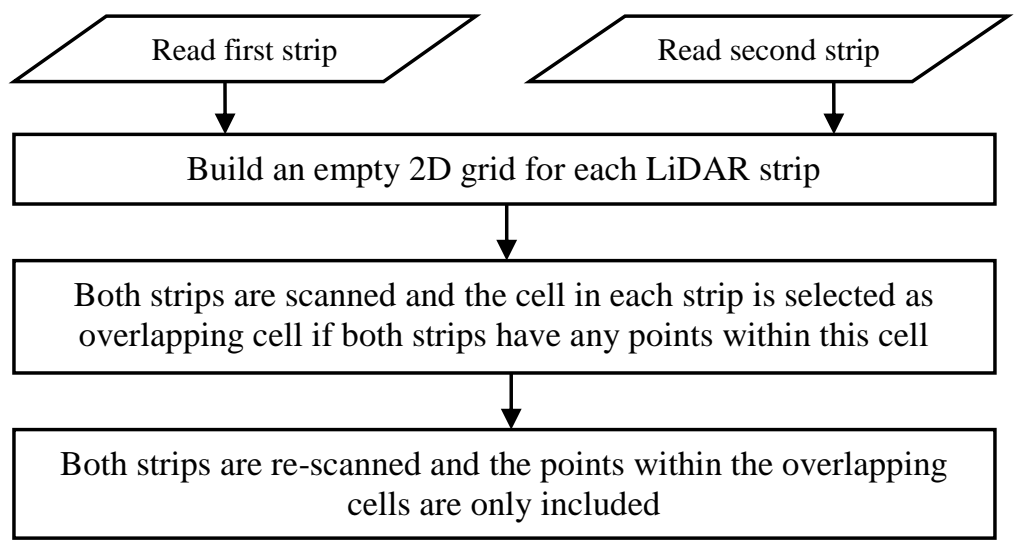

Figure 5. The flow chart for deriving the overlapping region between LiDAR Strips

\begin{tabular}{|c|c|c|c|c|c|c|c|c|c|}
\hline $\mathbf{x}$ & $\mathbf{x}$ & $\mathbf{x}$ & $\mathbf{x}$ & $\mathbf{x}$ & $\mathbf{x}$ & $\mathbf{x}$ & $\mathbf{x}$ & $\mathbf{x}$ & $\mathbf{x}$ \\
\hline $\mathbf{x}$ & $\mathbf{x}$ & $\mathbf{x}$ & $\mathbf{x}$ & $\boldsymbol{x}$ & $\mathbf{x}$ & $\mathbf{x}$ & $\mathbf{x}$ & $\mathbf{x}$ & $\mathbf{x}$ \\
\hline $\mathbf{x}$ & $\mathbf{x}$ & $\mathbf{x}$ & $\mathbf{x}$ & $\boldsymbol{v}$ & $\boldsymbol{v}$ & $\boldsymbol{x}$ & $\mathbf{x}$ & $\mathbf{x}$ & $\mathbf{x}$ \\
\hline $\mathbf{x}$ & $\mathbf{x}$ & $\mathbf{x}$ & $\mathbf{x}$ & $\boldsymbol{v}$ & $\boldsymbol{v}$ & $\boldsymbol{v}$ & $\mathbf{x}$ & $\mathbf{x}$ & $\mathbf{x}$ \\
\hline $\mathbf{x}$ & $\mathbf{x}$ & $\mathbf{x}$ & $\mathbf{x}$ & $\boldsymbol{v}$ & $\boldsymbol{v}$ & $\boldsymbol{v}$ & $\boldsymbol{y}$ & $\mathbf{x}$ & $\mathbf{x}$ \\
\hline $\mathbf{x}$ & $\mathbf{x}$ & $\mathbf{x}$ & $\mathbf{x}$ & $\boldsymbol{v}$ & $\boldsymbol{v}$ & $\boldsymbol{v}$ & $\mathbf{y}$ & $\mathbf{x}$ & $\mathbf{x}$ \\
\hline $\mathbf{x}$ & $\mathbf{x}$ & $\mathbf{x}$ & $\mathbf{x}$ & $\mathbf{x}$ & $\boldsymbol{v}$ & $\boldsymbol{v}$ & $\mathbf{x}$ & $\mathbf{x}$ & $\mathbf{x}$ \\
\hline $\mathbf{x}$ & $\mathbf{x}$ & $\mathbf{x}$ & $\mathbf{x}$ & $\mathbf{x}$ & $\mathbf{x}$ & $\mathbf{x}$ & $\mathbf{x}$ & $\mathbf{x}$ & $\mathbf{x}$ \\
\hline
\end{tabular}

Figure 6. The selected overlapping region between two LiDAR strips $(\sqrt{ })$

Overlap 2

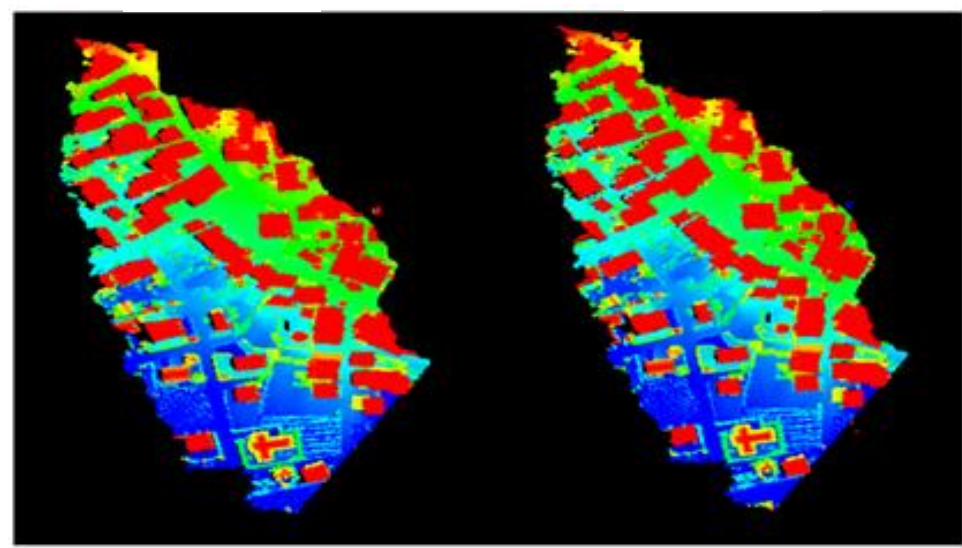

Figure 7. Visualization of the overlapping region between two LiDAR strips 


\subsubsection{Automatic identification of appropriate overlapping strip pairs from each group}

From the previous step, all possible overlapping regions between LiDAR strip pairs in each category are identified. One might ask, what is the criterion to select the most suitable overlapping strip pairs which should achieve the minimum optimal flight configuration (two overlapping strip pairs flown in different flying heights in opposite directions and one overlapping strip pair flown in parallel direction) to be used in the calibration procedure?

The number of points in the overlapping region as a measure or criterion to choose the suitable overlapping strip pairs is not enough since some of the necessary slope and aspect angles for a balanced distribution are not represented by the clustered regions. Additionally, the number of clustered regions in the overlapping area is also not sufficient as a measure or criterion to select the appropriate overlapping strip pairs because slope and aspect angles of some clustered regions don't cover the required slope and aspect range for accurate calibration. As mentioned before, regions with varying slope and aspect angles within the overlapping strips can be used as suitable regions to represent the LiDAR surfaces. In this step, in each category, we are interested in segmenting one of the overlapping strip pairs into homogenous clustered regions. The applied segmentation process is the one proposed by Lari et al., 2011. Figure 8 shows sample of segmented clusters within one strip from a given pair (related to the illustrated strips in Figure 7) that include gable roofs with varying slope and aspect.

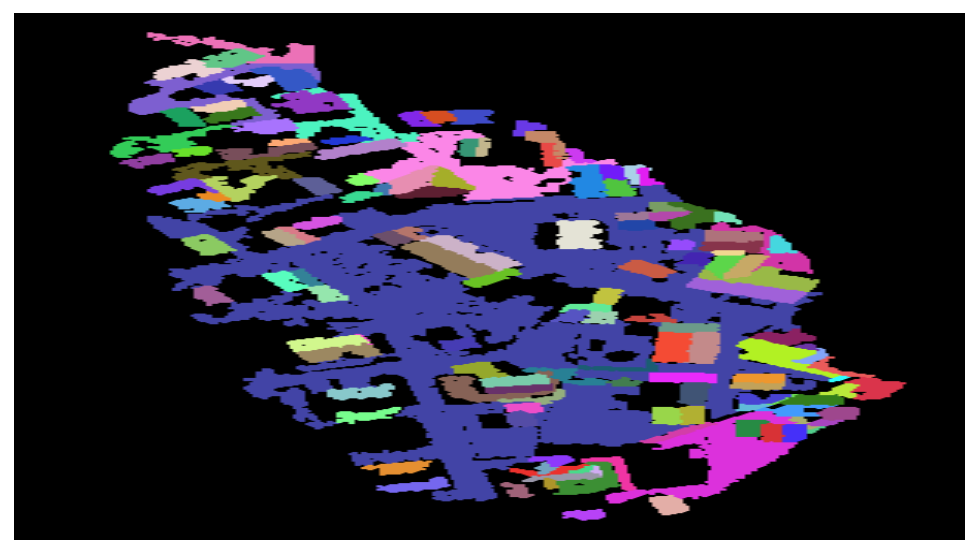

Figure 8. Visualization of segmented clusters within one overlapping strip from a given pair that include some gable roofs with varying slope and aspect

The proposed method in this research work establishes the variations in slope and aspect angles (angular coverage) of clustered regions within overlapping strip pairs as a suitable measure to select the appropriate overlapping strip pairs to be used in LiDAR system calibration. Figure 9 illustrates the flowchart for deriving an estimate of the angular coverage. First, angular coverage grid is created with pre-defined cell dimensions (Slope_Step, Aspect_Step) to represent the covered slope and aspect angles of each clustered region in the overlapping area as shown in Figure 10. From the segmentation process, the surface normal component $\left(\mathrm{n}_{\mathrm{x}}, \mathrm{n}_{\mathrm{y}}, \mathrm{n}_{\mathrm{z}}\right)$ of each clustered region is calculated and then, the slope $(\alpha)$ and aspect $(\theta)$ for each given clustered region is computed as shown in Figure 11. The corresponding angular coverage aspect and slope indices $\left(\mathrm{C}_{-} \mathrm{i}, \mathrm{C}_{-} \mathrm{j}\right)$ for each clustered region are calculated as in Equation 2, 3. The cell at this index is labeled as covered cell $(\sqrt{ })$ as shown in Figure 10. The previous steps are repeated until all clustered regions are investigated. Finally, the angular coverage is calculated as in Equation 4. 


$$
\begin{gathered}
C_{-} i=(\theta+\pi) \times 180 /(\pi \times \text { Aspect_Step }) \\
C_{-j}=(\alpha+\pi / 2) \times 180 /(\pi \times \text { Slope_Step })
\end{gathered}
$$

angular coverage $=\frac{\text { Number of non-empty cells in the angular coverage grid }}{\text { Total number of all cells in the angular coverage grid }} \times 100$

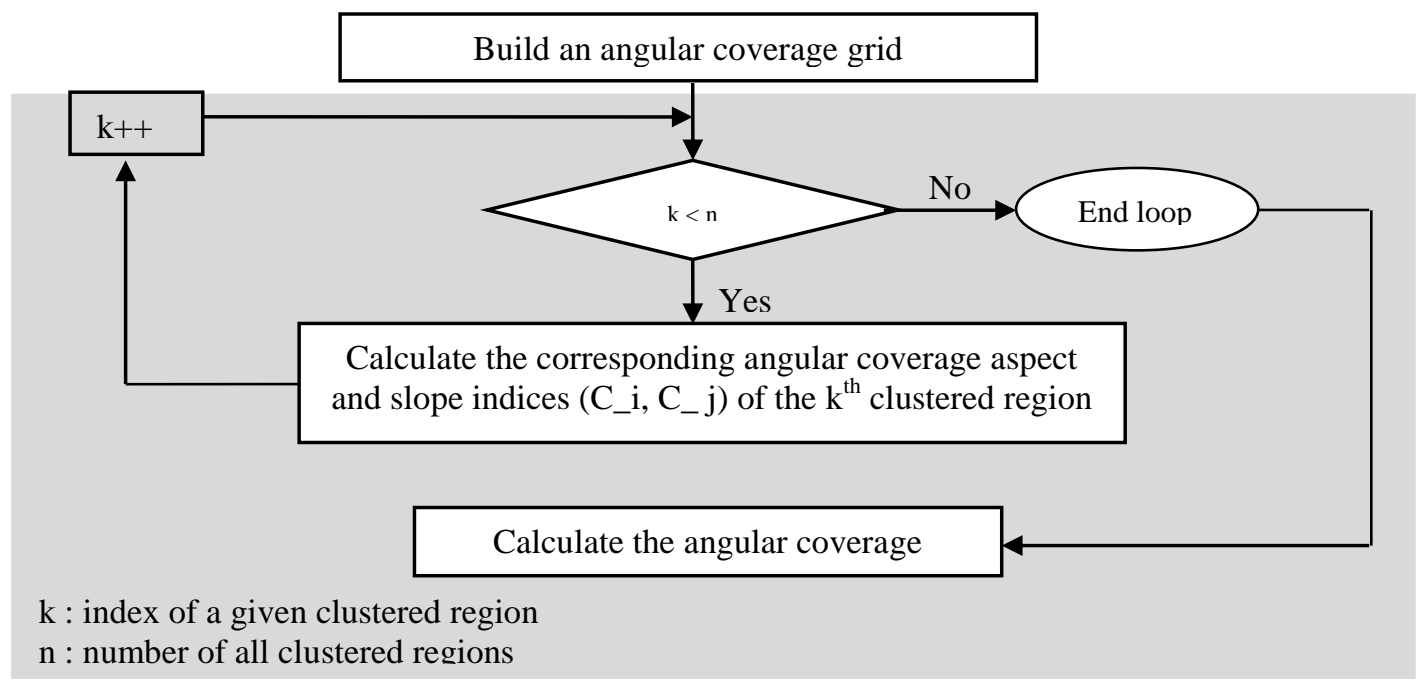

Figure 9. The flow chart for computing the angular coverage

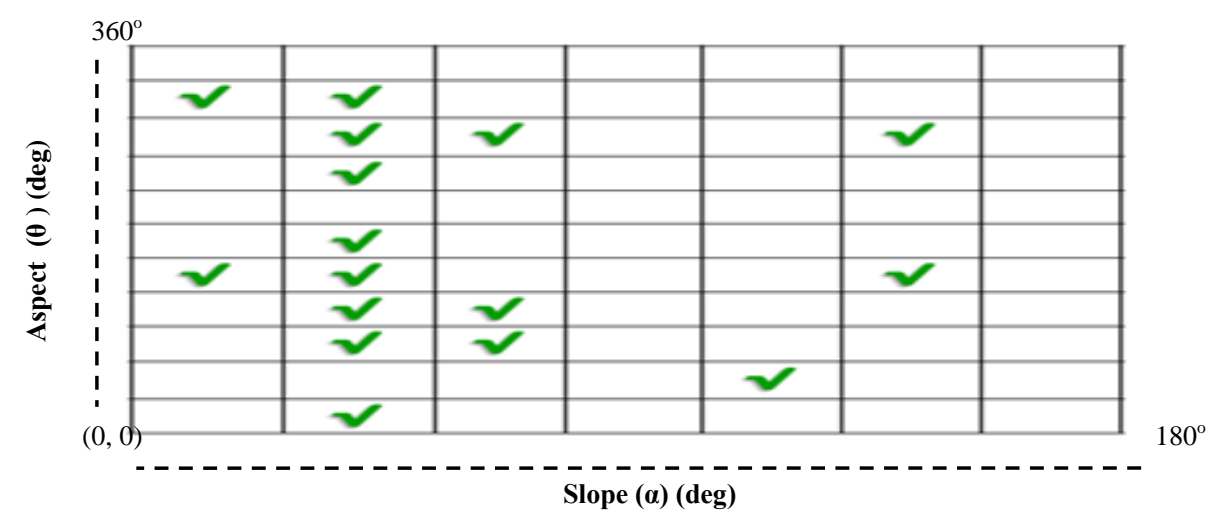

Figure 10. The grid representation of the covered slope/aspect angles by the segmented clusters

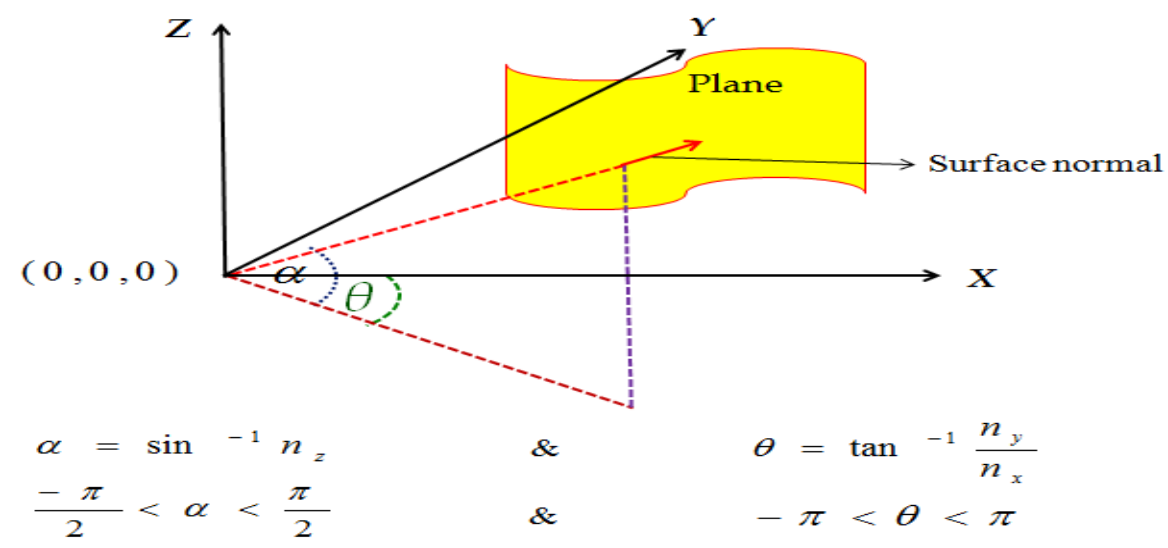

Figure 11. The evaluation of the slope and aspect angles for a given cluster 
The angular coverage percentages are calculated for all possible overlapping strip pairs in each category. We are interested in achieving the minimum optimal flight configuration which includes opposite/parallel groups of LiDAR strip pairs, so the cross groups in each category are excluded (as presented in Table 3). The answer of the pervious question is the overlapping strip pair with high angular coverage percentage in each group is selected to be used in the calibration procedure. In case of two or more overlapping strip pairs have the same angular coverage percentages (refer to overlapping strip pairs (2\&4), and (3\&9)), the overlapping strip pair with high overlap percentage is selected to be used in the calibration procedure. From Table 3, one can deduce that the selected overlapping strip pairs form each group are (2\&4), (1\&9), and (5\&7).

Table 3. Samples of overlapping strip pairs based on angular coverage percentage

\begin{tabular}{c|c|c|c|c|c}
\hline \hline $\begin{array}{c}\text { First } \\
\text { strip }\end{array}$ & $\begin{array}{c}\text { Second } \\
\text { strip }\end{array}$ & $\begin{array}{c}\text { Category based on } \\
\text { flying height }\end{array}$ & Flight Direction & $\begin{array}{c}\text { Overlap } \\
\text { Percentage } \\
(\%)\end{array}$ & $\begin{array}{c}\text { Angular } \\
\text { coverage } \\
\text { Percentage } \\
(\%)\end{array}$ \\
\hline \hline 1 & 9 & & Parallel Direction & $75 \%$ & $13 \%$ \\
\hline 1 & 3 & \multirow{2}{*}{ Category 1 } & Opposite Direction & $22 \%$ & $5 \%$ \\
\hline 2 & 4 & & Opposite Direction & $42 \%$ & $8 \%$ \\
\hline 3 & 9 & & Opposite Direction & $40 \%$ & $8 \%$ \\
\hline 5 & 7 & \multirow{2}{*}{ Category 2 } & Opposite Direction & $64 \%$ & $11 \%$ \\
\cline { 5 - 6 } & 8 & Opposite Direction & $60 \%$ & $8 \%$ \\
\hline \hline
\end{tabular}

\subsection{Automatic Selection of Regions within the Selected Overlapping Strip Pairs}

From the previous steps, the suitable overlapping strip pairs are selected. The objective of this step is to ensure the quality of the estimated parameters and increase the computational speed of the calibration process by reducing the number of clustered regions among the overlapping strip pairs, while maintaining the candidate clustered regions with good variations in slope, aspect, and covered range over the whole overlapping area as much as possible. The proposed technique is based on following two steps:

- Selection of candidate clustered regions with good variations in slope and aspect angles,

- Selection of candidate clustered regions that should be well-distributed within the whole overlapping area.

\subsubsection{Selection of the clustered regions based on their slopes and aspects}

The purpose of this step is to minimize the number of clustered regions by removing the clustered regions with redundant slope and aspect angles while maintaining the clustered regions with large size (maximum number of points). The method of selecting the candidate clustered region with good variations in slope and aspect angles can be summarized as follows: first, from the previous steps that were discussed in Figure 9, the covered slope and aspect angles of each clustered regions in the overlapping area is represented in the angular coverage grid. Then, for each covered cell in the angular coverage grid, all clustered regions inside this cell are checked and the clustered region with maximum size (maximum number of points) is selected as a candidate region and one should add it to the list of possible candidate clustered regions. Figure 12 shows sample of automatically selected clustered regions based on the slope and aspect criterion (related to the illustrated strip in Figure 8). 


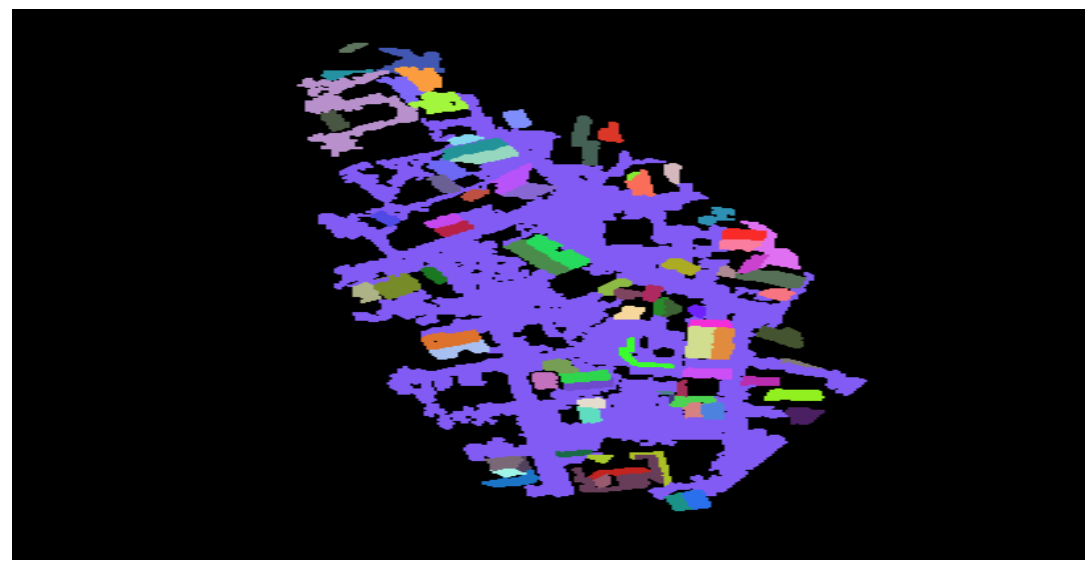

Figure 12. Visualization of automatically selected clustered regions based on the slope and aspect criterion

\subsubsection{Selection of appropriate clustered regions based on their distribution in the whole overlapping area}

The aim of this step is to ensure that all clustered regions are well-distributed over the whole overlapping area by adding new candidate clusters that are chosen by their distribution and are not chosen before by their slope and aspect angles. Moreover, we have to have a balance between the sizes of the different clustered regions. In other words, if one clustered region is excessively larger than the others, it will cause overweighting for a specific slope and aspect values when compared to the other clustered regions. Hence, the quality of the estimated parameters in the calibration procedure will be affected. The algorithm of selecting the candidate clustered regions based on their distribution over the whole overlapping area can be summarized as follows:

- First; an empty 2D grid is created to represent the extent of the overlapping area. This grid is divided into cells based on the dimensions of the overlapping area.

- Second; one can note from Figure 12 that the biggest clustered region is the ground and it is extended over the whole overlapping area. If the ground is showing good distribution of slope and aspect values, it should not be excluded. In the case that appears in Figure 12, we want to exclude the ground. The ground can be excluded as follows:

- All cells in the gird are checked and for each given cell, all clustered regions inside this cell are checked and for each given clustered region, if 50\% or more of their points are inside this cell, this region is kept. One can note that this constraint will ensure the ground is excluded because $50 \%$ or more of their points cannot completely be inside one cell in the grid.

- Then, all kept regions inside a given cell are checked and the region with the maximum ratio (number of points of a given region inside the cell divided by total number of points of a given region) is selected as a candidate region based on the distribution criterion.

- Finally; all candidates clustered regions based on the slope and aspect criterion are checked and if the candidate region based on the distribution criterion is found, then this region is already taken before (covered before based on the slope and aspect criterion). If the region is not chosen before based on the slope and aspect criterion, then this region should be selected as a candidate region based on the distribution criterion and one should add it to the list of possible candidate clustered regions. 
At the end, the list of possible candidate clustered regions holds the candidate regions based on the slope and aspect criterion, and the candidate regions based on the distribution criterion. Figure 13 shows sample of automatically selected clustered regions among overlapping strip pairs (related to the displayed clustered regions in Figure 12).

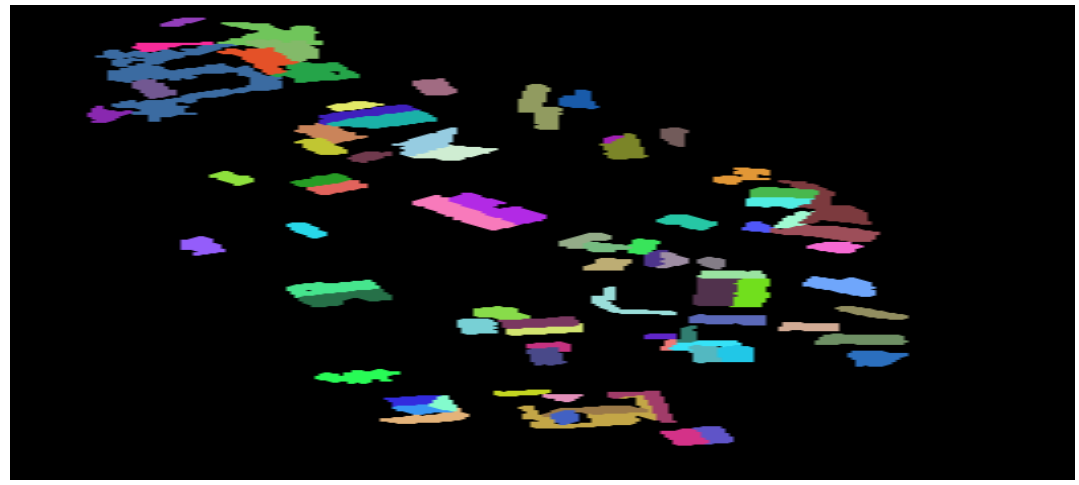

Figure 13. Visualization of automatically selected candidate clustered regions between two overlapping strips

Table 4 presents samples of automatically selected candidate regions with different slopes, aspects, and distribution among the selected overlapping strip pairs. From Table 4 one can deduce that the number of candidate clustered regions is reduced compared to the total number of clustered regions among the overlapping strip pairs while maintaining the representation of the candidate clustered regions with good variations in slope, aspect, and distribution.

Table 4. Samples of automatically selected clustered regions with different slopes, aspects, and distribution among the selected overlapping strip pairs

\begin{tabular}{c|c|c|c|c}
\hline \hline First strip & Second strip & Flight Direction & $\begin{array}{c}\text { Total number of } \\
\text { clustered regions }\end{array}$ & $\begin{array}{c}\text { Number of utilized } \\
\text { regions in the } \\
\text { calibration }\end{array}$ \\
\hline \hline 1 & 9 & Parallel Direction & 157 & 83 \\
\hline 2 & 4 & Opposite Direction & 117 & 55 \\
\hline 5 & 7 & Opposite Direction & 150 & 74 \\
\hline \hline
\end{tabular}

\section{Experimental Results}

The experimental results will be presented in this section to show the comparative analysis (i.e., quality of the estimated parameters, quality of fit between conjugate surfaces in overlapping strips, and the data processing speed) between manual and automatic selection of regions among overlapping LiDAR strips. This comparative analysis is carried out using the "Rigorous" calibration procedure proposed by Kersting, (2011). The experiments were carried out using a real dataset which is selected to evaluate the performance of the proposed method. This dataset includes airborne laser data collected in Switzerland with Scan2Map mapping system. Figure 14 shows the flight configuration of the LiDAR strips to be used in the calibration procedure. We study 2 cases (manual and automatic) for selecting the pairs/regions among the LiDAR strips to estimate the system parameters for 3 test scenarios: Test scenario "I" corresponds to the minimum optimal configuration, consisting of three overlapping pairs (two flown in different flying heights in opposite directions and one flown in parallel 
direction). Test scenario "II" adds one more overlapping pair to the minimum configuration, for a total of four overlapping pairs. Finally, test scenario "III" adds control data to the scenario "II". The objective of Test scenario "II" and "III" is evaluating the performance of the proposed calibration procedure by adding one more overlapping pair and control data to the minimum configuration. Table 5 presents the characteristics of involved overlapping strip pairs utilized in the calibration procedure using manual and automatic selection of regions among these overlapping strip pairs. The number of matched point-patch pairs in the calibration process using manual and automatic selection of regions among overlapping strip pairs is reported in Table 5. Table 6 shows the tested scenarios. Tables 7 and 8 report the estimated system parameters using Rigorous calibration method for the 3 test scenarios in case of manual and automatic selection of regions among overlapping strip pairs respectively.

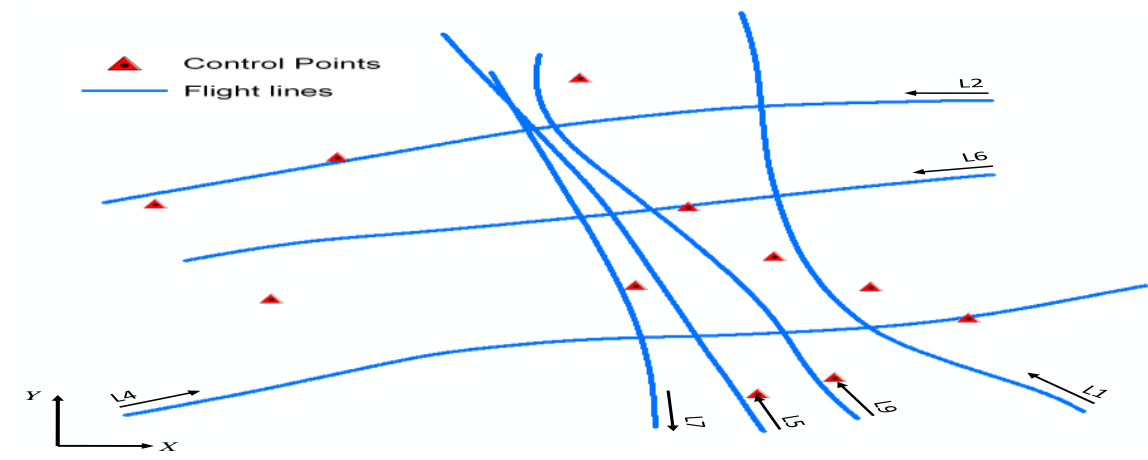

Figure 14. The flight configuration of LiDAR strips to be used in the calibration procedure

Table 5. Characteristics of the LiDAR overlapping strip pairs used in the calibration procedure

\begin{tabular}{|c|c|c|c|c|}
\hline Overlap Pair & $\begin{array}{c}\text { Flight } \\
\text { Configuration }\end{array}$ & $\begin{array}{c}\text { Flying } \\
\text { height (m) }\end{array}$ & $\begin{array}{c}\text { Number of Matched } \\
\text { Point-Patch Pairs out of } \\
\text { the total number of } \\
\text { Points in } S_{1} \text { using } \\
\text { manual selection } \\
\end{array}$ & $\begin{array}{c}\text { Number of Matched } \\
\text { Point-Patch Pairs out of } \\
\text { the total number of } \\
\text { Points in } S_{I} \text { using } \\
\text { automatic selection } \\
\end{array}$ \\
\hline (a) Strips $1 \& 9$ & Parallel direction & 699 & $\begin{array}{c}16545 \text { out of } \\
20608 \\
\end{array}$ & $\begin{array}{c}23820 \text { out of } \\
144656\end{array}$ \\
\hline (b) Strips $2 \& 4$ & opposite direction & 703 & $\begin{array}{c}8039 \text { out of } \\
11677\end{array}$ & $\begin{array}{c}12241 \text { out of } \\
85989\end{array}$ \\
\hline (c) Strips $5 \& 7$ & opposite direction & 795 & $\begin{array}{c}10783 \text { out of } \\
13690 \\
\end{array}$ & $\begin{array}{c}12240 \text { out of } \\
115688\end{array}$ \\
\hline (d) Strips 5\&6 & Cross direction & 804 & $\begin{array}{c}12842 \text { out of } \\
17123\end{array}$ & $\begin{array}{c}14726 \text { out of } \\
137985 \\
\end{array}$ \\
\hline
\end{tabular}

Table 6. List of overlapping strip pairs used for the tested scenarios.

\begin{tabular}{c|c}
\hline \hline Test Scenario & Overlapping Pairs \\
\hline \hline I & $($ a $),(b)$, and $(\mathrm{c})$ \\
\hline II & $($ a $),(b),(c)$, and $(d)$ \\
\hline III & $($ a $),(b),(c),(d)$ and Control Data \\
\hline
\end{tabular}


Table 5. Estimated system parameters using manual selection of regions among overlapping strip pairs

\begin{tabular}{|c|c|c|c|c|c|}
\hline & $\begin{array}{c}\Delta \omega \\
(\sec \pm \sec )\end{array}$ & $\begin{array}{c}\Delta \varphi \\
(\sec \pm \sec )\end{array}$ & $\begin{array}{c}\Delta \kappa \\
(\sec \pm \sec )\end{array}$ & $S$ & $\begin{array}{l}\text { Processing } \\
\text { time (sec) }\end{array}$ \\
\hline $\begin{array}{c}\text { Proposed Calibration } \\
\text { (Overlapping Strips Only: Scenario I) }\end{array}$ & $\begin{array}{l}-342.2 \\
\pm 0.5\end{array}$ & $\begin{array}{l}104.3 \\
\pm 0.7\end{array}$ & $\begin{array}{l}226.8 \\
\pm 2.7\end{array}$ & $\begin{array}{c}1.00009 \\
\pm 0.00001\end{array}$ & 170 \\
\hline $\begin{array}{c}\text { Proposed Calibration } \\
\text { (Overlapping Strips Only: Scenario II) }\end{array}$ & $\begin{array}{l}-336.9 \\
\pm 0.5\end{array}$ & $\begin{array}{c}114.7 \\
\pm 0.6\end{array}$ & $\begin{array}{l}230.0 \\
\pm 2.2\end{array}$ & $\begin{array}{c}1.00017 \\
\pm 0.00001\end{array}$ & 125 \\
\hline $\begin{array}{c}\text { Proposed Calibration } \\
\text { (Overlapping Strips + Control Data : } \\
\text { Scenario III) }\end{array}$ & $\begin{array}{l}-340.8 \\
\pm 0.5\end{array}$ & $\begin{array}{l}115.6 \\
\pm 0.6\end{array}$ & $\begin{array}{l}227.9 \\
\pm 2.2\end{array}$ & $\begin{array}{c}1.00005 \\
\pm 0.00001\end{array}$ & 172 \\
\hline
\end{tabular}

Table 6. Estimated system parameters using automatic selection of appropriate regions among overlapping strip pairs

\begin{tabular}{|c|c|c|c|c|c|}
\hline & $\begin{array}{c}\Delta \omega \\
(\sec \pm \sec )\end{array}$ & $\begin{array}{c}\Delta \varphi \\
(\sec \pm \sec )\end{array}$ & $\begin{array}{c}\Delta \kappa \\
(\sec \pm \sec )\end{array}$ & $S$ & $\begin{array}{l}\text { Processing } \\
\text { time (sec) }\end{array}$ \\
\hline $\begin{array}{c}\text { Proposed Calibration } \\
\text { (Overlapping Strips Only: Scenario I) }\end{array}$ & $\begin{array}{l}-332.9 \\
\pm 0.4\end{array}$ & $\begin{array}{l}103.5 \\
\pm 0.6\end{array}$ & $\begin{array}{c}237.6 \\
\pm 2.7\end{array}$ & $\begin{array}{r}1.00007 \\
\pm 0.00001\end{array}$ & 85 \\
\hline $\begin{array}{c}\text { Proposed Calibration } \\
\text { (Overlapping Strips Only: Scenario II) }\end{array}$ & $\begin{array}{l}-328.9 \\
\pm 0.4\end{array}$ & $\begin{array}{l}109.4 \\
\pm 0.6\end{array}$ & $\begin{array}{c}230.4 \\
\pm 2.4\end{array}$ & $\begin{array}{r}1.00024 \\
\pm 0.00001\end{array}$ & 90 \\
\hline $\begin{array}{c}\text { Proposed Calibration } \\
\text { (Overlapping Strips + Control Data : } \\
\text { Scenario III) }\end{array}$ & $\begin{array}{l}-331.2 \\
\pm 0.4\end{array}$ & $\begin{array}{l}110.3 \\
\pm 0.6\end{array}$ & $\begin{array}{l}224.6 \\
\pm 2.4\end{array}$ & $\begin{array}{l}1.00019 \\
\pm 0.00001\end{array}$ & 92 \\
\hline
\end{tabular}

To verify the quality of the estimated parameters, the discrepancies among conjugate surface elements in overlapping strips have been computed before and after the calibration process using the nominal and estimated system parameters, respectively. The utilized nominal values for the system parameters are $\Delta \omega=\Delta \varphi,=\Delta \rho=0, \Delta \mathrm{k}=90$, and $\boldsymbol{S}=1$. Tables 9 and 10 report the determined discrepancies between overlapping strip pairs before and after applying the calibration process for the 3 test scenarios in case of manual and automatic selection of regions among overlapping strip pairs respectively.

From Tables 9 and 10, we can observe large discrepancies among the overlapping strip pairs before the calibration procedure. For instance, the overlapping strip pair 2 and 4 (flown in opposite directions - east-west) has a large shift in the y-axis, which is approximately the across flight direction. This discrepancy can be attributed to large deviation between the nominal and estimated boresight roll angle, which mainly affects the across-flight direction, i.e., a constant shift across the flight direction and a rotation around the flight direction. Similarly, the overlapping strip pair 5 and 7 (also flown in opposite directions-north-south) has a large shift in the $\mathrm{x}$-axis, which is approximately the across flight direction. The impact of the boresight roll angle is larger for the overlapping strip pair 5 and 7 due to the fact that it was flown at higher flying height. One should note that for the overlapping strip pair 1 and 9, no significant discrepancy in the across flight direction is observed before the calibration process. This is due to the fact that for strips flown in the same direction, inaccurate boresight roll angle only causes a constant vertical shift between conjugate surface elements with a much smaller magnitude (the magnitude increases with an increased lateral distance between 
Table 7. Discrepancies (i.e., three shifts and three rotations) between overlapping strips before and after the calibration process (reconstructed using nominal and estimated system parameters) in manual selection

\begin{tabular}{|c|c|c|c|c|c|c|c|c|}
\hline \multicolumn{3}{|c|}{ Before Calibration } & \multicolumn{3}{|c|}{$\begin{array}{c}\text { Proposed Calibration } \\
\text { Overlapping Strips Only } \\
\text { (Scenario I / Scenario II) }\end{array}$} & \multicolumn{3}{|c|}{$\begin{array}{c}\text { Proposed Calibration } \\
\text { (Overlapping Strips + Control } \\
\text { Data : Scenario III) }\end{array}$} \\
\hline \multicolumn{9}{|c|}{$1 \& 9$} \\
\hline $\mathbf{X}_{\mathbf{T}}(\mathbf{m})$ & $\mathbf{Y}_{\mathrm{T}}(\mathbf{m})$ & $\overline{\mathbf{Z}_{\mathrm{T}}(\mathbf{m})}$ & $\mathbf{X}_{\mathbf{T}}(\mathbf{m})$ & $\mathbf{Y}_{\mathrm{T}}(\mathbf{m})$ & $\mathbf{Z}_{\mathbf{T}}(\mathbf{m})$ & $\mathbf{X}_{\mathrm{T}}(\mathbf{m})$ & $\mathbf{Y}_{\mathrm{T}}(\mathbf{m})$ & $\mathbf{Z}_{\mathbf{T}}(\mathbf{m})$ \\
\hline 0.0685 & -0.1989 & -0.0719 & $\begin{array}{c}0.0084 / \\
0.0163\end{array}$ & $\begin{array}{c}-0.0059 / \\
0.0012\end{array}$ & $\begin{array}{c}0.0152 / \\
0.0136\end{array}$ & 0.0173 & -0.004 & 0.0145 \\
\hline$\omega\left(^{0}\right)$ & $\varphi\left(\left(^{0}\right)\right.$ & $\kappa\left(\left(^{0}\right)\right.$ & $\omega\left(^{0}\right)$ & $\varphi\left({ }^{0}\right)$ & $\kappa\left(\left(^{0}\right)\right.$ & $\omega\left(^{0}\right)$ & $\varphi\left(\left(^{0}\right)\right.$ & $\kappa\left({ }^{0}\right)$ \\
\hline \multirow[t]{2}{*}{-0.0224} & 0.0098 & 0.0432 & $\begin{array}{c}\mathbf{0 . 0 1 3 9} / \\
0.0137\end{array}$ & $\begin{array}{c}0.014 / \\
0.0116\end{array}$ & $\begin{array}{c}0.0173 / \\
0.0155\end{array}$ & 0.0137 & 0.0128 & 0.0176 \\
\hline & & & & $2 \& 4$ & & & & \\
\hline $\mathbf{X}_{\mathbf{T}}(\mathbf{m})$ & $\mathbf{Y}_{\mathbf{T}}(\mathbf{m})$ & $\mathbf{Z}_{\mathbf{T}}(\mathbf{m})$ & $\mathbf{X}_{\mathrm{T}}(\mathbf{m})$ & $\mathbf{Y}_{\mathrm{T}}(\mathbf{m})$ & $\mathbf{Z}_{\mathbf{T}}(\mathbf{m})$ & $\mathbf{X}_{\mathrm{T}}(\mathbf{m})$ & $\mathbf{Y}_{\mathrm{T}}(\mathbf{m})$ & $\mathbf{Z}_{\mathbf{T}}(\mathbf{m})$ \\
\hline 0.0517 & 0.5642 & 0.0639 & $\begin{array}{r}-0.0167 / \\
-0.0367\end{array}$ & $\begin{array}{c}0.0614 / \\
0.0694\end{array}$ & $\begin{array}{l}-0.003 / \\
-0.0006\end{array}$ & -0.0402 & 0.0767 & -0.0013 \\
\hline$\omega\left(^{0}\right)$ & $\varphi\left({ }^{0}\right)$ & $\kappa\left(\left(^{0}\right)\right.$ & $\omega\left(^{0}\right)$ & $\varphi\left({ }^{0}\right)$ & $\kappa\left({ }^{0}\right)$ & $\omega\left(^{0}\right)$ & $\varphi\left({ }^{0}\right)$ & $\kappa\left({ }^{\mathbf{0}}\right)$ \\
\hline \multirow[t]{2}{*}{0.1408} & 0.0572 & 0.0008 & $\begin{array}{c}-0.0137 / \\
-0.0058\end{array}$ & $\begin{array}{c}0.021 / \\
0.0226\end{array}$ & $\begin{array}{c}-0.0081 / \\
-0.006\end{array}$ & -0.009 & 0.0223 & -0.0084 \\
\hline & & & & $5 \& 6$ & & & & \\
\hline $\mathbf{X}_{\mathbf{T}}(\mathbf{m})$ & $\mathbf{Y}_{\mathrm{T}}(\mathbf{m})$ & $\mathbf{Z}_{\mathbf{T}}(\mathbf{m})$ & $\mathbf{X}_{\mathrm{T}}(\mathbf{m})$ & $\mathbf{Y}_{\mathrm{T}}(\mathbf{m})$ & $\mathbf{Z}_{\mathbf{T}}(\mathbf{m})$ & $\mathbf{X}_{\mathrm{T}}(\mathbf{m})$ & $\mathbf{Y}_{\mathrm{T}}(\mathbf{m})$ & $\mathbf{Z}_{\mathrm{T}}(\mathbf{m})$ \\
\hline 0.3349 & -0.4631 & 0.0095 & $\begin{array}{c}-\mathbf{0 . 0 1 0 2 /} \\
\text { NA }\end{array}$ & $\begin{array}{c}-0.0184 / \\
\text { NA }\end{array}$ & $\begin{array}{c}\text { 0.0222/ } \\
\text { NA }\end{array}$ & -0.0114 & -0.0191 & 0.0227 \\
\hline$\omega\left(^{0}\right)$ & $\varphi\left(^{0}\right)$ & $\kappa\left(\left(^{0}\right)\right.$ & $\omega\left(^{0}\right)$ & $\varphi\left(\left(^{0}\right)\right.$ & $\kappa\left(\left(^{0}\right)\right.$ & $\omega\left(^{0}\right)$ & $\varphi\left({ }^{0}\right)$ & $\kappa\left({ }^{0}\right)$ \\
\hline \multirow[t]{2}{*}{-0.0626} & -0.1021 & -0.0083 & $\begin{array}{c}0.0011 / \\
\text { NA }\end{array}$ & $\begin{array}{c}\mathbf{0 . 0 0 6 9 /} \\
\text { NA }\end{array}$ & $\begin{array}{c}\text { 0.0018/ } \\
\text { NA }\end{array}$ & 0.0018 & 0.0078 & 0.0019 \\
\hline & & & & $5 \& 7$ & & & & \\
\hline $\mathbf{X}_{\mathbf{T}}(\mathbf{m})$ & $\mathbf{Y}_{\mathrm{T}}(\mathbf{m})$ & $\mathbf{Z}_{\mathbf{T}}(\mathbf{m})$ & $\mathbf{X}_{\mathrm{T}}(\mathbf{m})$ & $\mathbf{Y}_{\mathrm{T}}(\mathbf{m})$ & $\mathbf{Z}_{\mathbf{T}}(\mathbf{m})$ & $\mathbf{X}_{\mathrm{T}}(\mathbf{m})$ & $\mathbf{Y}_{\mathrm{T}}(\mathbf{m})$ & $\mathbf{Z}_{\mathrm{T}}(\mathbf{m})$ \\
\hline 0.7591 & -0.1461 & 0.0961 & $\begin{array}{c}0.0068 / \\
0.0127\end{array}$ & $\begin{array}{c}-0.0152 / \\
0.0058\end{array}$ & $\begin{array}{l}0.025 / \\
0.027\end{array}$ & 0.0115 & 0.0071 & 0.027 \\
\hline$\omega\left(^{0}\right)$ & $\varphi\left(^{0}\right)$ & $\kappa\left(\left(^{0}\right)\right.$ & $\omega\left(^{0}\right)$ & $\varphi\left(^{0}\right)$ & $\kappa\left(\left(^{0}\right)\right.$ & $\omega\left(\left(^{0}\right)\right.$ & $\varphi\left({ }^{0}\right)$ & $\kappa\left({ }^{\mathbf{0}}\right)$ \\
\hline 0.0832 & -0.1677 & 0.0032 & $\begin{array}{c}0.0033 / \\
0.0028\end{array}$ & $\begin{array}{c}0.0158 / \\
0.0135\end{array}$ & $\begin{array}{c}0.0223 / \\
0.0229\end{array}$ & 0.0024 & 0.0141 & 0.0229 \\
\hline
\end{tabular}

the strips - which is not the case for this strip pair). The slightly larger shift in the y-axis for the strip pair 1 and 9, which is approximately along the flight direction of these strips, can be attributed to an inaccurate nominal value for the boresight yaw angle. Similar to the other strip pairs, a significant improvement after the calibration process is noticeable.

\section{Conclusions and Recommendations Future Work}

In this paper a new approach was presented for automatic selection of appropriate overlapping strip pairs/regions, which should achieve the minimum optimal flight configuration to be used in the LiDAR system calibration. The experimental results have shown that the quality of the estimated parameters using the automatic selection are quite similar to the estimated parameters using the manual selection while the processing time of automatic selection is 2 times faster than the manual selection. These results prove that accurate estimation of the calibration parameters and faster data processing speed can be obtained using the proposed method. The contributions of the proposed method can be summarized as follows: 
Table 8. Discrepancies (i.e., three shifts and three rotations) between overlapping strips before and after the calibration process (reconstructed using nominal and estimated system parameters) in automatic selection

\begin{tabular}{|c|c|c|c|c|c|c|c|c|}
\hline \multicolumn{3}{|c|}{ Before Calibration } & \multicolumn{3}{|c|}{$\begin{array}{c}\text { Proposed Calibration } \\
\text { Overlapping Strips Only } \\
\text { (Scenario I / Scenario II) } \\
\end{array}$} & \multicolumn{3}{|c|}{$\begin{array}{c}\text { Proposed Calibration } \\
\text { (Overlapping Strips + Control Data } \\
\text { : Scenario III) }\end{array}$} \\
\hline \multicolumn{9}{|c|}{$1 \& 9$} \\
\hline $\mathbf{X T}(\mathbf{m})$ & $\mathbf{Y T}(\mathbf{m})$ & $\mathbf{Z T}(\mathbf{m})$ & $\mathrm{XT}(\mathrm{m})$ & $\mathbf{Y T}(\mathbf{m})$ & ZT(m) & $\mathbf{X T}(\mathbf{m})$ & YT(m) & ZT(m) \\
\hline 0.0619 & -0.1751 & -0.0664 & $\begin{array}{c}0.0045 / \\
0.0140\end{array}$ & $\begin{array}{c}-0.0204 / \\
-0.0163\end{array}$ & $\begin{array}{l}0.0081 / \\
0.0060\end{array}$ & 0.0131 & -0.0179 & 0.0066 \\
\hline$\omega(0)$ & $\varphi(\mathbf{0})$ & $\kappa(\mathbf{o})$ & $\omega(0)$ & $\varphi(0)$ & $\kappa(\mathbf{o})$ & $\omega(0)$ & $\varphi(\mathbf{0})$ & $\kappa(\mathbf{o})$ \\
\hline-0.0112 & 0.0115 & 0.0257 & $\begin{array}{c}0.0049 / \\
0.0060\end{array}$ & $\begin{array}{c}0.0203 / \\
0.0146\end{array}$ & $\begin{array}{c}0.0240 / \\
0.0221\end{array}$ & 0.0059 & 0.0161 & $\mathbf{0 . 0 2 3 0}$ \\
\hline \multicolumn{9}{|c|}{$2 \& 4$} \\
\hline $\mathbf{X T}(\mathbf{m})$ & $\mathbf{Y T}(\mathbf{m})$ & ZT(m) & $\mathrm{XT}(\mathrm{m})$ & $\mathbf{Y T}(\mathbf{m})$ & ZT(m) & $\mathbf{X T}(\mathrm{m})$ & YT(m) & ZT(m) \\
\hline $\begin{array}{c}-0.0690 \\
\omega(0)\end{array}$ & $\begin{array}{c}-0.6106 \\
\varphi(0)\end{array}$ & $\begin{array}{c}-0.0689 \\
\kappa(0)\end{array}$ & $\begin{array}{c}0.0309 / \\
0.0381 \\
\omega(0)\end{array}$ & $\begin{array}{c}-0.0973 / \\
-0.0950 \\
\varphi(0)\end{array}$ & $\begin{array}{l}0.0008 / \\
-0.0025 \\
\kappa(0)\end{array}$ & $\begin{array}{c}0.0341 \\
\omega(0)\end{array}$ & $\begin{array}{c}-0.0983 \\
\varphi(0)\end{array}$ & $\begin{array}{c}-0.0013 \\
\kappa(0)\end{array}$ \\
\hline-0.1765 & -0.0553 & 0.0114 & $\begin{array}{c}-0.0113 / \\
-0.0244\end{array}$ & $\begin{array}{l}-0.0137 / \\
-0.0169\end{array}$ & $\begin{array}{c}0.0201 / \\
0.0186\end{array}$ & -0.0201 & -0.0158 & 0.0164 \\
\hline \multicolumn{9}{|c|}{$5 \& 6$} \\
\hline $\mathbf{X T}(\mathbf{m})$ & $\mathbf{Y T}(\mathbf{m})$ & ZT(m) & $\mathbf{X T}(\mathbf{m})$ & YT(m) & ZT(m) & $\mathbf{X T}(\mathrm{m})$ & $\mathbf{Y T}(\mathbf{m})$ & ZT(m) \\
\hline-0.3219 & 0.4668 & $-\mathbf{- 0 . 0 2 3 3}$ & $\begin{array}{c}0.0030 / \\
\text { NA }\end{array}$ & $\begin{array}{c}\text { 0.0110/ } \\
\text { NA }\end{array}$ & $\begin{array}{c}-0.0289 / \\
\text { NA }\end{array}$ & 0.0046 & 0.0085 & -0.0297 \\
\hline$\omega(0)$ & $\varphi(\mathbf{0})$ & $\kappa(\mathbf{0})$ & $\omega(0)$ & $\varphi(\mathbf{o})$ & $\kappa(0)$ & $\omega(0)$ & $\varphi(\mathbf{0})$ & $\kappa(\mathbf{0})$ \\
\hline 0.0627 & 0.1029 & 0.0296 & $\begin{array}{c}0.0027 / \\
\text { NA }\end{array}$ & $\begin{array}{c}-0.0044 / \\
\text { NA }\end{array}$ & $\begin{array}{c}0.0070 / \\
\text { NA }\end{array}$ & 0.0021 & -0.0056 & 0.0054 \\
\hline \multicolumn{9}{|c|}{$5 \& 7$} \\
\hline $\mathbf{X T}(\mathbf{m})$ & $\mathbf{Y T}(\mathbf{m})$ & ZT(m) & XT(m) & YT(m) & ZT(m) & $\mathrm{XT}(\mathbf{m})$ & $\mathbf{Y T}(\mathbf{m})$ & ZT(m) \\
\hline 0.7379 & -0.1190 & 0.0500 & $\begin{array}{c}.0006 / \\
-0.0021\end{array}$ & $\begin{array}{c}-0.0096 / \\
0.0041\end{array}$ & $\begin{array}{l}0.0220 / \\
0.0230\end{array}$ & -0.0042 & 0.0068 & 0.0228 \\
\hline$\omega(0)$ & $\varphi(0)$ & $\kappa(\mathbf{o})$ & $\omega(0)$ & $\varphi(\mathbf{o})$ & $\kappa(\mathbf{o})$ & $\omega(0)$ & $\varphi(0)$ & $\kappa(\mathbf{o})$ \\
\hline 0.0737 & -0.1728 & -0.0006 & $\begin{array}{c}0.0078 / \\
0.0075\end{array}$ & $\begin{array}{c}0.0078 / \\
0.0074\end{array}$ & $\begin{array}{c}0.0164 / \\
0.0148\end{array}$ & 0.0072 & 0.0079 & 0.0157 \\
\hline
\end{tabular}

- The proposed method is fully automated,

- The proposed method doesn't depend on the operator, while the manual selection of overlapping pairs/regions proposed by Kersting, (2011) relies on the experience of the operator,

- Appropriate overlapping strip pairs which should achieve the minimum optimum flight configuration are automatically selected. Also, regions which represent suitable LiDAR surfaces (regions with varying slope, aspect, and distribution within the overlapping strips) to be used in the calibration procedure are automatically selected compared to the manual selection of overlapping pairs/regions proposed by Kersting, (2011),

- The proposed method leads to accurate estimation of the calibration parameters, and

- The proposed method is used for faster and reliable LiDAR system calibration. 
Future research work will focus on the possibility of extending existing calibration techniques to deal with multi-scanner LiDAR systems and the possibility of using the proposed procedure for calibrating terrestrial mobile laser scanning systems.

\section{Acknowledgement}

The authors would like to thank TECTERRA for the financial support of this research work.

\section{References}

[1] Ackermann, F., 1999. Airborne laser scanning - present status and future expectations, ISPRS Journal of Photogrammetry and Remote Sensing, Vol. 54, No. 2-3, pp. 64-67.

[2] Ayman Habib \& Jim Van RensQuality.,2009. Assurance and Quality Control of LiDAR Systems and Derived Data., ASPRS PAD Lidar Committee (ASPRS LC)

[3] Bang, K.I., Habib, A.F., Kersting, A., 2010. Estimation of Biases in LiDAR System Calibration Parameters Using Overlapping Strips, The Canadian Journal of Remote Sensing, (accepted 2010).

[4] Csanyi, N., 2008. A Rigorous Approach to Comprehensive Performance Analysis of State-of-the-Art Airborne Mobile Mapping Systems, Ph.D. dissertation, The Ohio State University, $217 \mathrm{p}$.

[5] Habib, A. F., Bang, K.I., Shin, S.W., and Mitishita, E., 2007. LiDAR system selfcalibration using planar patches from photogrammetric data, The 5th International Symposium on Mobile Mapping Technology, [CD-ROM]. 28-31 May, Padua, Italy.

[6] Habib, A. F., Bang, K.I., Kersting, A., and Lee D.C., 2009a. Error budget of LiDAR systems and quality control of the derived data, Photogrammetric Engineering and Remote Sensing, Vol. 75, No. 9, pp. 1093-1108.

[7] Katzenbeisser, R., 2003. About the calibration of LiDAR sensors, Proceedings of the ISPRS working group III/3 workshop, "3-D Reconstruction from Airborne Laserscanner and InSAR Data", [CD-ROM]. 8-10 October, Dresden, Germany.

[8] Kersting, A. P., Habib, A., Bang, K. I., and Skaloud, J. Automated approach for rigorous light detection and ranging system calibration without preprocessing and strict terrain coverage requirements, Optical Engineering 51(7), 076201(July 2012)

[9] Kersting, A. P., "Quality assurance of multi-sensor systems," PhD dissertation, Dept. Geomatics Eng., Calgary, AB, p. 265, University of Calgary (2011).

[10] Lari, Z., Habib, A. and E. Kwak (2011). ."An Adaptive Approach for Segmentation of 3D Laser Point Cloud",ISPRS Workshop Laser Scanning

[11] 2011 Calgary, Canada 29 - 31 August 2011.

[12] Lee, J., Yu, K., Kim, Y., and Habib, A.F., 2007. Adjustment of Discrepancies between LiDAR Data Strips Using Linear Features, IEEE Geoscience and Remote Sensing Letter, Vol. 4, No. 3, pp. 475-479.

[13] Skaloud, J. and Schaer, P., 2007. Towards automated LiDAR boresight self-calibration, The 5th International Symposium on Mobile Mapping Technology, [CD-ROM]. 28-31 May, Padua, Italy. 